\title{
IKKa-mediated biogenesis of miR-196a through interaction with Drosha regulates the sensitivity of cancer cells to radiotherapy
}

\author{
X Fang ${ }^{1,2}$, J-H Jeong ${ }^{2}$, X Long ${ }^{1,2}$, S-J Park ${ }^{2}$, D Wang ${ }^{2}$, M Shuai ${ }^{1,2}$, R Wei ${ }^{1,2}$, C Li ${ }^{1}$, S Li ${ }^{1}$, S Zhang ${ }^{1}$, MB Duran ${ }^{2}$, K-W Lo ${ }^{3}$, \\ SW Tsao ${ }^{4}$, R Glaser ${ }^{5}$, Z Luo ${ }^{6}$, X Feng $^{*, 1,2}$, Y Tian ${ }^{\star, 1}$ and J-L Luo ${ }^{*, 1,2}$
}

\begin{abstract}
Radioresistance is a major obstacle in successful clinical cancer radiotherapy, and the underlying mechanisms are not clear. Here we show that IKK $\alpha$-mediated miR-196a biogenesis via interaction with Drosha regulates the sensitivity of nasopharyngeal carcinoma (NPC) cells to radiotherapy. Phosphorylation of IKK $\alpha$ at T23 site (p-IKK $\alpha$ T23) promotes the binding of IKK $\alpha$ to Drosha that accelerates the processing of miR-196a primary transcripts, leading to increased expressions of both precursor and mature miR-196a. Dephosphorylation of p-IKK $\alpha$ T23 downregulates miR-196a expression and promotes the resistance of NPC cells to radiation treatment. The miR-196a mimic suppresses while its inhibitor promotes the resistance of NPC to radiation treatment. Importantly, the expression of p-IKK $\alpha \mathrm{T} 23$ is positively related to the expression of miR-196a in human NPC tissues, and expression of $\mathrm{p}-\mathrm{IKK} \alpha \mathrm{T} 23$ and miR-196a is inversely correlated with NPC clinical radioresistance. Thus, our studies establish a novel mechanistic link between the inactivation of IKK $\alpha$ T23-Drosha-miR-196a pathway and NPC radioresistance, and de-inactivation of IKKoT23-Drosha-miR-196a pathway would be an efficient way to restore the sensitivity of radioresistant NPC to radiotherapy. Cell Death and Differentiation advance online publication, 8 April 2016; doi:10.1038/cdd.2016.32
\end{abstract}

Nasopharyngeal carcinoma (NPC) is one of the most common lethal malignancies diagnosed in Southeast Asia and southern China, where its incidence rate is approximately $25-50$ cases per 100000 population. ${ }^{1}$ About $90 \%$ of NPC are undifferentiated carcinoma. Radiation therapy is the primary treatment for undifferentiated NPC. Although some NPCs are sensitive to radiotherapy, some are de novo or develop resistance to radiotherapy. It has been reported that approximately $30 \%$ of patients presenting localized tumors develop recurrent disease, and $30-60 \%$ of patients with metastatic NPC die within 5 years of diagnosis. ${ }^{2,3}$ The mechanisms that cause NPC radiotherapy resistance are elusive. ${ }^{4}$

$\mathrm{IKK} a$ is a subunit of $\mathrm{I}_{K} \mathrm{~B}$ kinase (IKK) complex that consists of two highly homologous kinase subunits (IKKa and IKK $\beta$ ) and a nonenzymatic regulatory component, IKK $/$ NEMO $^{5}$ Nuclear factor $-\kappa \mathrm{B}(\mathrm{NF}-\kappa \mathrm{B})$ is generally activated through IKKdependent phosphorylation and subsequent degradation of $I_{\kappa} B$ inhibitory proteins. ${ }^{6}$ Liberated NF- $\kappa$ B dimers then enter the nucleus where they induce the transcription of various genes. ${ }^{7,8}$ There are two NF-KB activation pathways: the classical pathway ${ }^{6,9}$ and the alternative pathway. ${ }^{10-12}$ IKKa contributes to the control of both pathways and functions in the cytoplasm. Recently, we found that IKKa can be translocated into the nucleus and nuclear IKKa plays important roles in prostate cancer progression and metastasis. ${ }^{13,14}$

MicroRNAs (miRNAs) regulate gene expression through suppression of translation and decay of target mRNAs, and are involved in diverse physiological and pathological processes. The primary transcripts of miRNA (pri-miRNA) are cleaved into precursor miRNA (pre-miRNA) by nuclear Drosha, and further processed to mature miRNAs by cytoplasmic Dicer1 in mammalian miRNA biogenesis. The Drosha complex consists of Drosha, DGCR8 (DiGeorge syndrome critical region gene 8), DDX5 (RNA helicase p68) and DDX17 (RNA helicase p72). ${ }^{15}$ Recently, it has been reported that Smads, p53 and breast cancer 1 (BRCA1) are involved in miRNA maturation. ${ }^{16-18}$

Here, we examined the role of IKKa in cancer radiotherapy resistance, and found that IKKa-mediated biogenesis of miR-196a via interaction with Drosha regulates the sensitivity of NPC cells to radiation treatment.

\section{Results}

IKKa is dephosphorylated at $\mathrm{T} 23$ site in radioresistant NPC cells. To investigate the mechanisms that cause NPC

\footnotetext{
${ }^{1}$ Xiangya Hospital, Central South University, Hunan 410008, China; ${ }^{2}$ Department of Cancer Biology, The Scripps Research Institute, Jupiter, FL 33458 , USA; ${ }^{3}$ Department of Anatomical \& Cellular Pathology, Faculty of Medicine, The Chinese University of Hong Kong, Hong Kong SAR, China; ${ }^{4}$ Department of Anatomy, Li Ka Shing Faculty of Medicine, The University of Hong Kong, Hong Kong SAR, China; ${ }^{5}$ Department of Molecular Virology, Immunology, and Medical Genetics, Institute for Behavioral Medicine Research, The Ohio State University, Columbus, OH, USA and ${ }^{6}$ School of Life Sciences, Central South University, Hunan 410008, China

${ }^{*}$ Corresponding author: J-L Luo or X Feng or Y Tian, Xiangya Hospital, Central South University, or Department of Cancer Biology, The Scripps Research Institute, Florida Campus, USA, 130 Scripps Way, Jupiter, FL 33458, USA. Tel: +1 561 2283202; E-mail: jlluo@scripps.edu or fxp1029@aliyun.cn or yongquantian2013@163.com Abbreviations: ATM, ataxia telangiectasia-mutated protein kinase; BRCA1, Breast cancer susceptibility gene 1; CHK2, Checkpoint kinase 2; DDX5, DEAD (Asp-Glu-AlaAsp) box helicase 5; DDX17, DEAD-box helicase 17; DHX9, DEAH (Asp-Glu-Ala-His) Box Helicase 9; DGCR8, DiGeorge syndrome critical region gene 8; EMSA, electrophoretic mobility shift assay; hnRNPA1, heterogeneous nuclear ribonucleoprotein A1; IKK, IKB kinase; IR, ionizing radiation; NF- $\kappa$ B, nuclear factor kappa-light-chainenhancer of activated B cells; NPC, nasopharyngeal carcinoma; PI3K, phosphoinositide 3-kinase; RIP, RNA immunoprecipitation; Smad, Sma and Mad Received 14.7.15; revised 22.2.16; accepted 29.2.16; Edited by V Stambolic
} 
radioresistance, we established several radioresistant NPC cell lines (Figure 1a) via two different approaches. The radioresistant C666-S8GY cells were obtained from the colonies survived from those C666-1 NPC cells treated with a single high dosage of radiation ( $8 \mathrm{~Gy}$ ). The radioresistant C666-M2GY cells were from those survived C666-1 NPC cells treated with radiation 2 Gy a day, 5 days a week for four consecutive weeks. To confirm the radioresistance of these cell lines, C666-S8GY, C666-M2GY and control C666-1 cells were treated with 6 Gy radiation, and cell viability, soft agar colony formation and tumor sphere formation were determined. We found that both C666-S8GY and C666-M2GY a

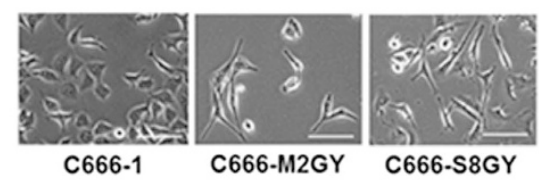

C

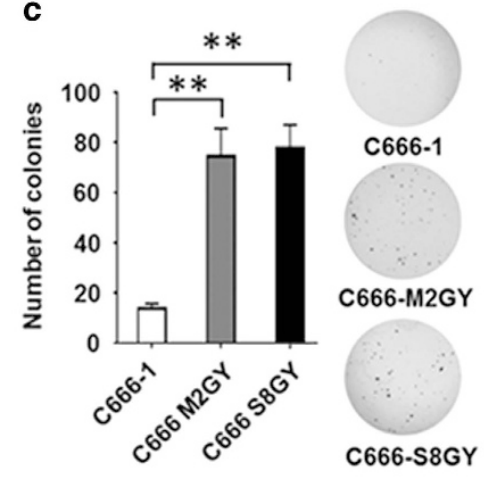

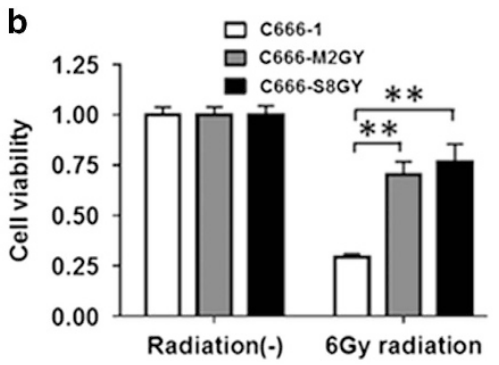

d C666-1 C666-M2GY C666-S8GY

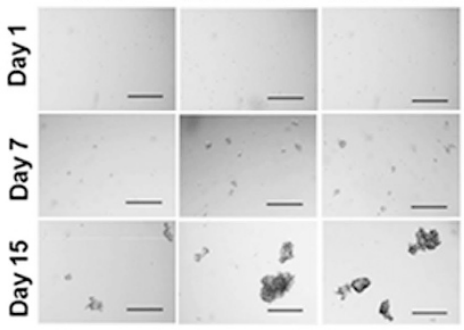

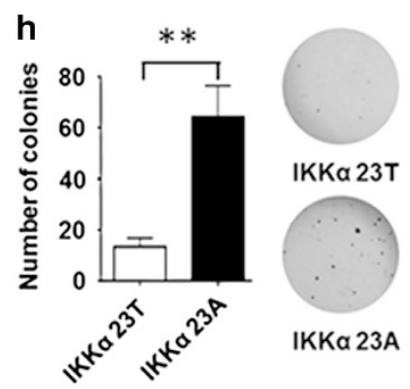
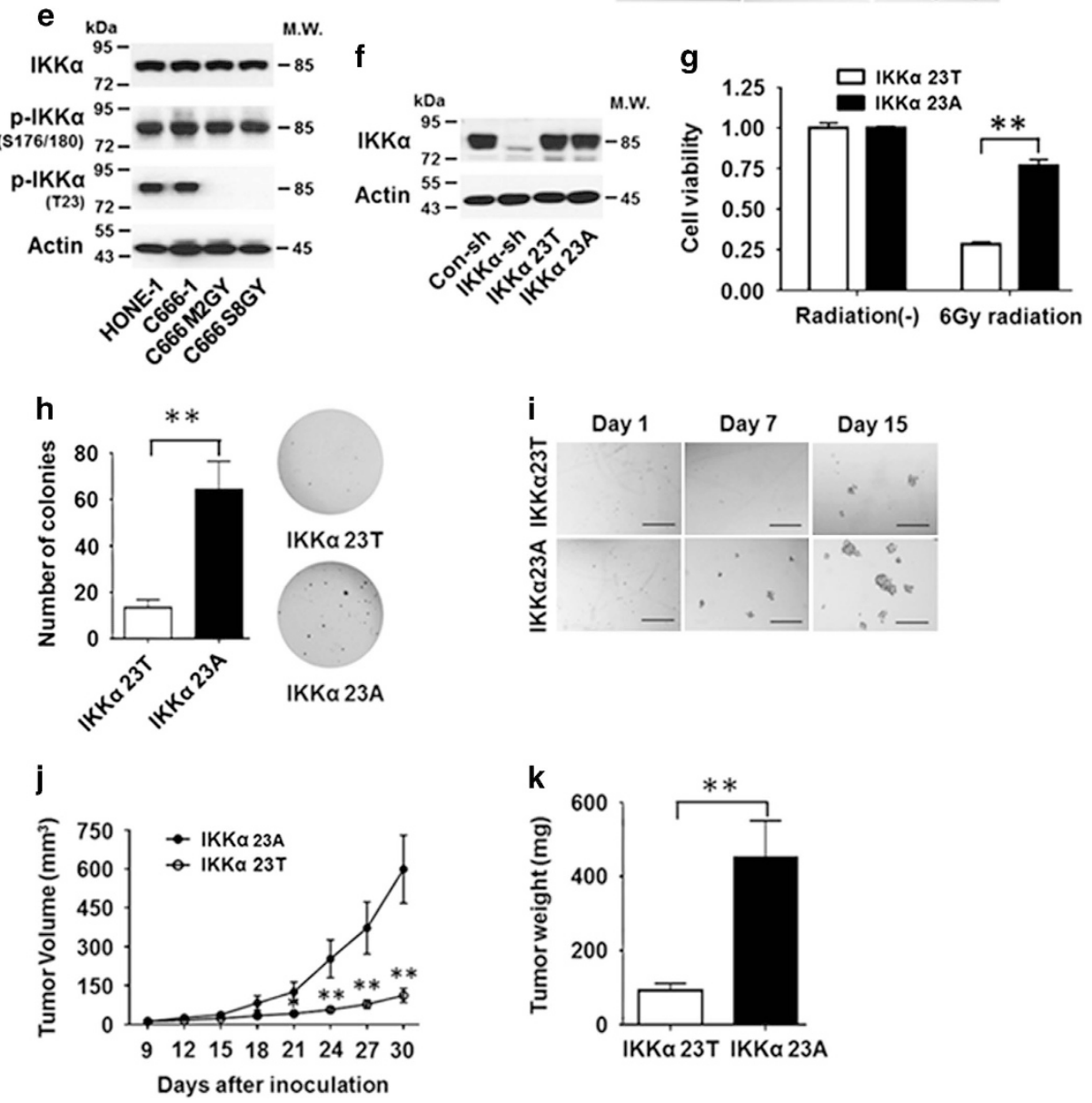
cells were highly resistant to radiation treatment (Figure 1b). In addition, the irradiated C666-S8GY and C666-M2GY cells formed much more colonies in soft agar and had much bigger tumor spheres in suspension culture than irradiated control C666-1 cells (Figures 1c and d).

It has been reported that IKKa plays a role in NPC development. ${ }^{19,20}$ To examine whether IKKa is activated in radioresistant NPC cells, the phosphorylation levels of IKKa at S176/S180 and T23 sites were detected by western blot. Surprisingly, we found that the expression level of p-IKKaT23 in both C666-S8GY and C666-M2GY cells was significantly decreased while the phosphorylation of IKKa at S176/S180 sites was unchanged as compared with C666-1 control cells (Figure 1e). Using the same strategy as for C666-1 cell, we established radioresistant HONE1 cells. Similarly, we found that the expression level of p-IKKaT23 in radioresistant HONE1 cells was significantly decreased while the phosphorylation of IKKa at $\mathrm{S} 176 / \mathrm{S} 180$ sites was unchanged as compared with HONE1 control cells. These results indicate that IKKa is dephosphorylated at T23 site in radioresistant NPC cells.

The dephosphorylation of p-IKKaT23 promotes NPC cell radioresistance. To investigate whether the phosphorylation of IKKaT23 is related to the sensitivity of NPC cells to radiation, we established an IKKa stable knocked-down cell line (C666-IKKa-KD) by infecting C666-1 cells with IKKa shRNA lentivirus that specifically target $3^{\prime}$-UTR of IKKa. C666-IKKa-KD cells were used to express exogenous IKKa as IKKa shRNA does not interfere with open reading frames expression of IKKa. We established stable cell lines by transfecting C666-IKKa-KD cells with plasmid expressing HA-tagged wild type or a mutant IKKa where the 23rd amino acid threonine was replaced by alanine through mutagenesis. These cell lines were named C666-IKKa-23A cells and C666-IKKa-23T cells, and expressed similar amounts of exogenous IKKa protein to the endogenous IKKa protein of C666-1 control cells (Figure 1f). MTT assay showed that C666-IKKa-23A cells were more resistant to radiation than C666-IKKa-23T cells (Figure 1g). C666-IKKa23A cells pretreated with 6 Gy of radiation had more colony formation in soft agar and formed more and bigger tumor spheres in suspension culture than C666-IKKa-23T cells pretreated with the same dosage of radiation (Figures $1 \mathrm{~h}$ and i). Furthermore, we found that C666-IKKa-23A cells pretreated with $6 \mathrm{~Gy}$ of radiation developed xenograft tumors in $\mathrm{Rag}^{-1-}$ mice considerably more rapidly than the preirradiated C666-IKKa-23T cells (Figures 1j and k). These results suggest that the dephosphorylation of p-IKKaT23 promotes NPC cells resistance to radiation treatment.

IKKaT23 regulates precursor and mature miRNA expression. In a campaign for the role of IKKa in prostate cancer development by microarray and quantitative RT-PCR(qRTPCR), we found that a set of precursor and mature miRNAs, including miR-196a, miR-494 and miR-615, was downregulated whereas their primary transcripts were unchanged in IKKa knocked-down Myc-CaP cells (Figure 2a). We confirmed that the downregulation of these precursor and mature miRNAs was also shown in C666-IKKa-KD cells (Figure 2b). Furthermore, we found that these precursor and mature miRNAs were also decreased in C666-IKKa-23A as compared with C666-IKKa-23T cells (Figure 2c), suggesting that the regulation of IKKa on the precursor and mature miR-196a, miR-494 and miR-615 is related to the phosphorylation status of IKKaT23. Supporting this, the precursor and mature miR-196a, miR-494 and miR-615 were upregulated in another NPC cell line HONE1, while their expression levels were very low in NP69, a immortalized normal nasopharyngeal epithelial cell line in which IKKaT23 was not phosphorylated (Supplementary Figure S1). Importantly, these precursor and mature miRNAs were also downregulated in C666-S8GY, C666-M2GY and radioresitant HONE1 cells, among them miR-196a was the most decreased one in radioresistant NPC cells (Figure 2d). Therefore, in the following studies we focused on the characterization and the role of miR-196a in IKKa23 T-associated radioresistance.

IKKaT23 mediates pri-miR-196a processing via interaction with drosha complex. The precursor and mature miR-196a were downregulated whereas its primary transcripts were unchanged in IKKa knocked-down cells, suggesting that IKKa may regulate miRNA biogenesis at the precursor level. As Drosha controls the biogenesis of miRNA precursors, the requirement of IKKa for miR-196a processing could be via its interaction with the Drosha microprocessor complex. To determine whether IKKa interacts with Drosha, we did co-immunoprecipitation between endogenous IKKa and Drosha in both C666-1 and Myc-CaP cells, and found that IKKa and Drosha could be reciprocally co-immunoprecipitated in both cells (Figure $3 a$ and Supplementary Figure S2). We also did co-immunoprecipitation with anti-HA antibody in C666-IKKa-23T and C666-IKKa-23A cells, and found that HA-IKKa23 T could pull down Drosha while HA-IKKa23 A could not co-immunoprecipitate Drosha (Figure 3b), suggesting the phosphorylation status of IKKa at T23 is associated

Figure 1 The KK $\alpha$ T23 phosphorylation status is related to the sensitivity of NPC cells to radiation treatment. (a) Establish radioresistant cell lines (C666-S8GY and C666M2GY cells) using two different approaches. Scale bars represent $100 \mu \mathrm{m}$. (b) MTT assay shows cell viability of C666-S8GY, C666-M2GY and control C666-1 cells after radiation treatment. (c) Soft agar colony formation of C666-S8GY, C666-M2GY and control C666-1 cells after 6 Gy radiation treatment. (d) Tumor sphere formation of C666-S8GY, C666M2GY and control C666-1 cells after 6 Gy radiation treatment. Scale bars represent $100 \mu \mathrm{m}$. (e) Western blot analysis for the expression of p-IKK $\alpha \mathrm{T} 23$ and p-IKK $\alpha \mathrm{S} 176 / \mathrm{S} 180$ in indicated NPC cell lines. (f) Western blot analysis for the expression of IKK $\alpha$ in C666-IKK $\alpha$-23A (IKK $\alpha 23 \mathrm{~A})$, C666-IKK $\alpha$-23T (IKK $\alpha 23 \mathrm{~T})$, C666-IKK $\alpha$-KD (IKK $\alpha$-sh) and control C666-1 (Con-sh) cell lines. (g) MTT assay shows cell viability of C666-IKK $\alpha-23 \mathrm{~A}$ and C666-IKK $\alpha$-23T cells after different dosages of radiation treatment. (h) Soft agar colony formation of C666-IKK $\alpha$-23A and C666-IKK $\alpha$-23T cells after 6 Gy radiation treatment. (i) Tumor spheres formation of C666-IKK $\alpha$-23A and C666-IKK $\alpha$-23T cells after 6 Gy of radiation treatment. Scale bars represent $100 \mu \mathrm{m}$. (j) Tumorigenecity analysis in Rag $1^{-l}$ - mice inoculated with C666-IKK $\alpha$-23T (IKK $\left.\alpha 23 \mathrm{~T}\right)$ or C666-IKK $\alpha$-23A (IKK $\left.\alpha 23 \mathrm{~A}\right)$ cells pretreated with 6 Gy of radiation treatment. (k) Comparison of the weight of tumors derived from irradiated C666-IKK $\alpha$-23T (IKK $\alpha 23 \mathrm{~T})$ and C666-IKK $\alpha$-23A (IKK $\alpha 23 \mathrm{~A})$ cells inoculated in Rag ${ }^{-1-}$ mice for 30 days. Note: ${ }^{* *} P<0.01$ 

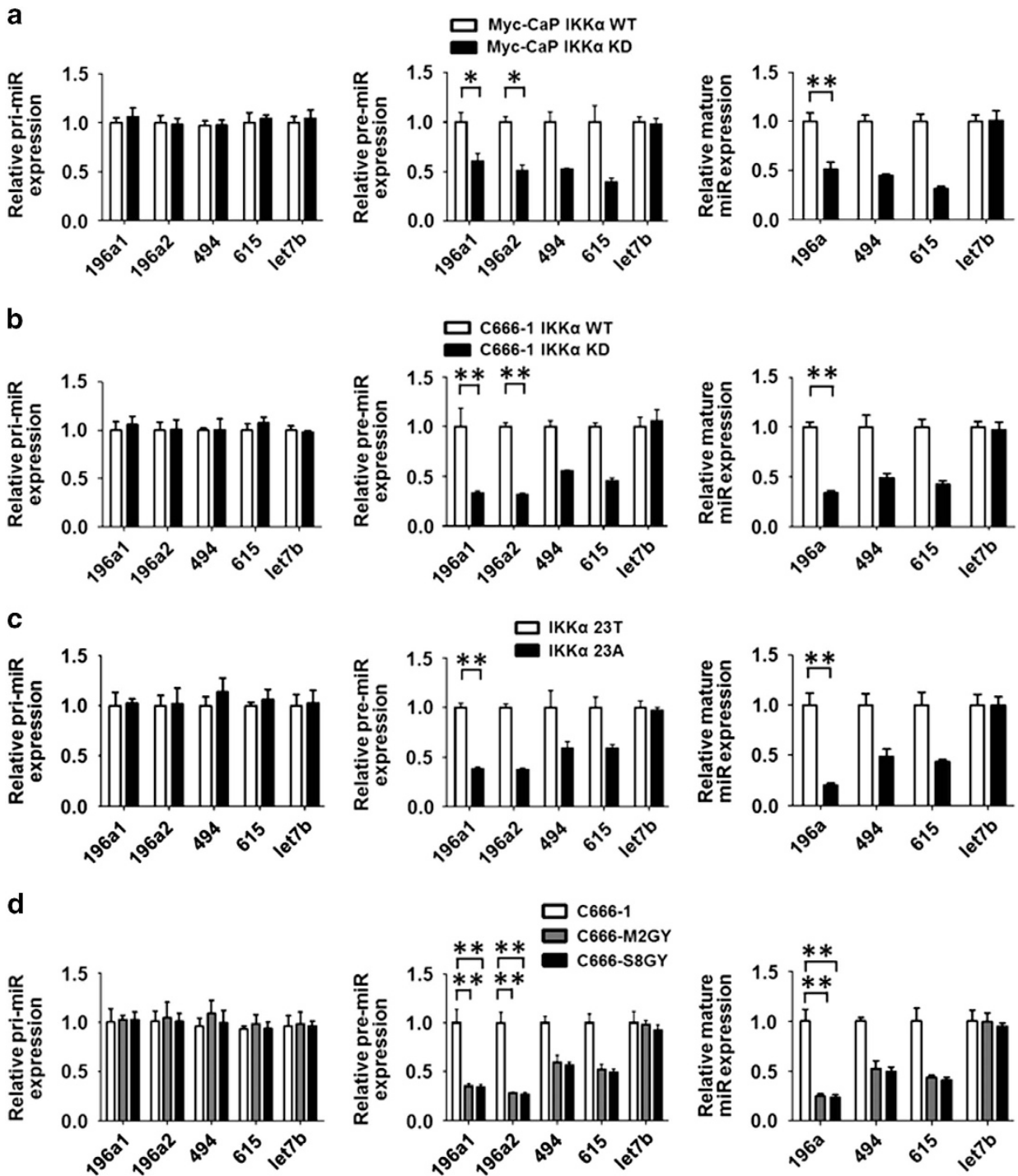

Figure 2 IKK $\alpha$ T23 regulates the expression of pre- and mature miRNAs. (a) Expression levels of the primary (pri), precursor (pre) and mature forms of the indicated miRNAs were examined in IKK $\alpha$ knockout and control Myc-CaP prostate cancer cells using gRT-PCR analysis. (b) Expression levels of the primary (pri), precursor (pre) and mature forms of the indicated miRNAs were examined in C666-IKK $\alpha$-KD and control C666-1 NPC cells using qRT-PCR analysis. (c) Expression levels of the primary (pri), precursor (pre) and mature (mat) forms of the indicated miRNAs were examined in C666-IKK $\alpha-23 \mathrm{~A}(\mathrm{IKK} \alpha 23 \mathrm{~A})$ and C666-IKK $\alpha-23 \mathrm{~T}$ (IKK $\alpha 23 \mathrm{~T})$ cells using qRT-PCR analysis. (d) Expression levels of the primary (pri), precursor (pre) and mature forms of the indicated miRNAs were examined in C666-S8GY, C666-M2GY and control C666-1 cells using qRT-PCR analysis. Note: Pri- and pre-miRNAs were normalized by GAPDH, and mature miRNA was normalized by U6 snRNA ( $\left.P<0.05 ;{ }^{\star \star} P<0.01 ; n=3\right)$

with the interaction between IKKa and Drosha. Supporting this hypothesis, IKKa and Drosha could be reciprocally co-immunoprecipitated in C666-1 cells, while the IKKa antibody could not or co-immunoprecipitated much lower level of Drosha in both radioresistant NPC cell lines, C666-S8GY and C666-M2GY, in which IKKa was dephosphorylated at T23 site (Figure 3c). Together, these results suggest that phosphorylation of IKKa23 T promotes the binding of IKKa protein to the Drosha complex.

To determine whether IKKa processes pri-miR-196a substrate, we performed an in vitro pri-miRNA processing assay by incubating FITC-labeled pri-let-7b, pri-miR-196a1 and pri-miR-196a2 substrates with immunoprecipitated IKKa complex from C666-IKKa-23T and C666-IKKa-23A cells.
Immunoprecipitated IKKa complex from C666-IKKa-23T cells potentiated the processing of pri-miR-196a1, pri-miR-196a2, but not pri-let-7b, whereas immunoprecipitated IKKa complex from C666-IKKa-23A cells did not potentiate the processing of pri-miR-196a1, pri-miR-196a2 and pri-let-7b (Figure 3d), indicating that immunoprecipitated IKKa-23T complex has pri-miRNA processing activity while the IKKa-23A complex lost this function. Supporting this hypothesis, immunoprecipitated IKKa complex from C666-1 cells potentiated the processing of pri-miR-196a1 and pri-miR-196a2, while immunoprecipitated IKKa complex from C666-S8GY and C666-M2GY cells, where IKKa-T23 was dephosphorylated, did not potentiate the processing of pri-miR-196a1 and pri-miR-196a2 (Figure 3e). 
a

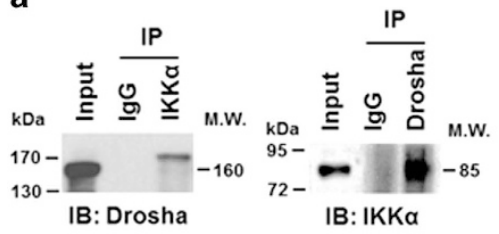

d

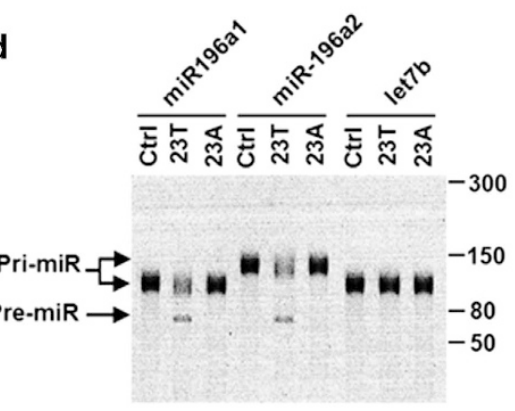

f

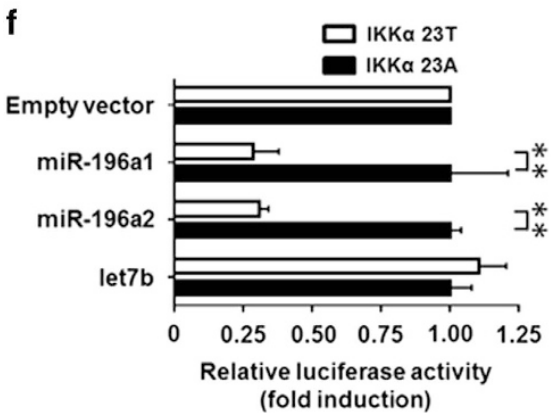

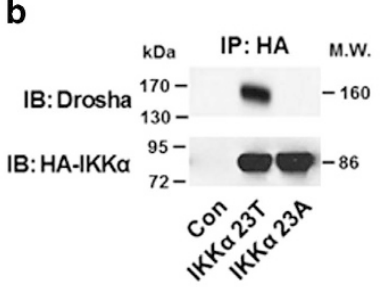

C

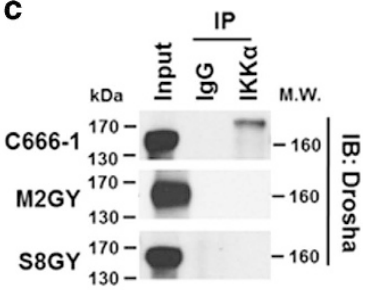

C666-1 C666-M2GY C666-S8GY

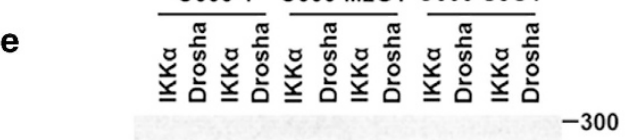

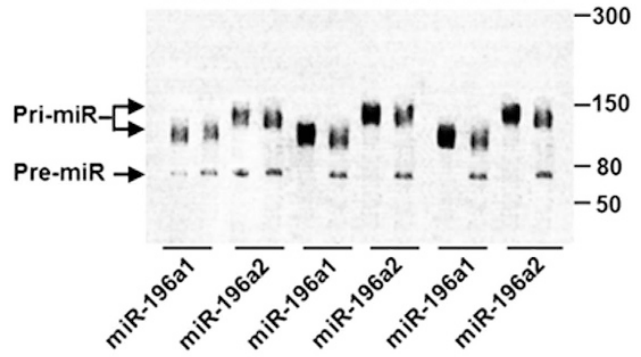
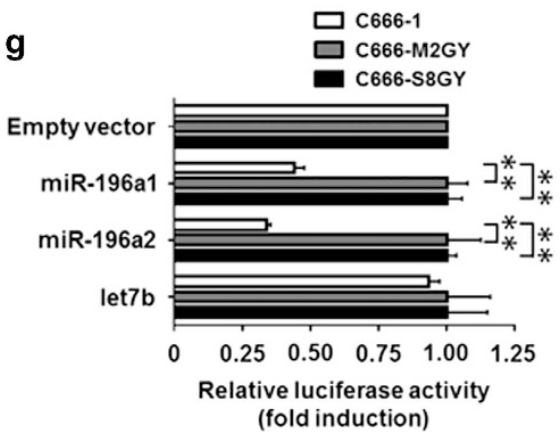

Figure 3 IKK $\alpha$ mediates pri-miR-196a processing by interacting with the Drosha complex. (a) C666-1 NPC cells were immunoprecipitated (IP) with anti-IKK $\alpha$ or anti-Drosha, followed by immunostaining with anti-IKK $\alpha$ or anti-Drosha antibody. (b) C666-IKK $\alpha-23 \mathrm{~A}$ and C666-IKK $\alpha-23 \mathrm{~T}$ cells were IP with anti-HA, followed by immunostaining with the antiDrosha antibody or anti-HA antibody. (c) C666-S8GY cells (S8GY), C666-M2GY cells (M2GY) and control C666-1 cells were IP with anti-IKK $\alpha$, followed by immunostaining with the anti-Drosha antibody. (d) In vitro pri-miRNA processing assays of pri-miR-196a1, pri-miR-196a2 with IKK $\alpha$ or Drosha complex immunoprecipitated from C666-IKK $\alpha$-23A and C666-IKK $\alpha$-23T cells. (e) In vitro pri-miRNA processing assays of pri-miR-196a1 and pri-miR-196a2 with immunoprecipitated IKK $\alpha$ or Drosha complex from C666-S8GY, C666M2GY and control C666-1 cells. (f) In vivo monitoring assay of pri-miRNA processing in C666-IKK $\alpha$-KD and C666-1 control cells carrying pri-miR-196a1 and pri-miR-196a2 at the $3^{\prime}$ untranslated region of the luciferase gene. The intensities were normalized by renilla luciferase and are shown as fold induction as compared with an empty vector $\left({ }^{* *} P<0.01\right.$; $n=3$ ). (g) In vivo monitoring assay of pri-miRNA processing in C666-S8GY, C666-M2GY and control C666-1 cells carrying pri-miR-196a1 and pri-miR-196a2 at the $3^{\prime}$ untranslated region of the luciferase gene. The intensities were normalized by renilla luciferase and are shown as fold induction as compared with an empty vector

Furthermore, we also conducted an in vivo cellular monitoring assay. C666-IKKa-23T and C666-IKKa-23A cells were transfected with a luciferase construct carrying a segment of pri-miR-196a1, pri-miR-196a2 or pri-let-7b between the luciferase gene and the polyadenylation signal. If luciferase transcripts lose their polyadenylation tail due to the cleavage of pri-miRNAs, its stability and translation efficiency will be significantly decreased. Consistent with the in vitro results, we found that the luciferase activity in C666IKKa-23T cells transfected with luciferase construct carrying a segment of miR-196a1, pri-miR-196a2 in 3'-UTR was significantly decreased, while it was unchanged in C666IKKa-23A cells (Figure 3f). In addition, we found that that the luciferase activity in C666-1 control cells transfected with luciferase construct carrying a segment of miR-196a1, primiR-196a2, in 3'-UTR was significantly decreased, while it was unchanged in C666-S8GY and C666-M2GY cells where IKKaT23 was dephosphorylated (Figure 3g). Collectively, these results demonstrate that the phosphorylation status of IKKa at T23 site is important for the binding of IKKa protein to the Drosha complex and consequently for the processing of primary miR-196a.

IKKa binds to primary miR-196a. Our studies showed IKKaT23 only regulated the processing of a group of miRNAs (Figure 2). To investigate why IKKaT23 specifically processes the biogenesis of primary miR-196a a co-immunoprecipitation between endogenous IKKa and Drosha in C666-1 cells was performed, followed by addition of RNase A. We found that the association of IKKa with Drosha was markedly decreased by treatment with RNase A, indicating that IKKa interacts with the Drosha complex via RNA 


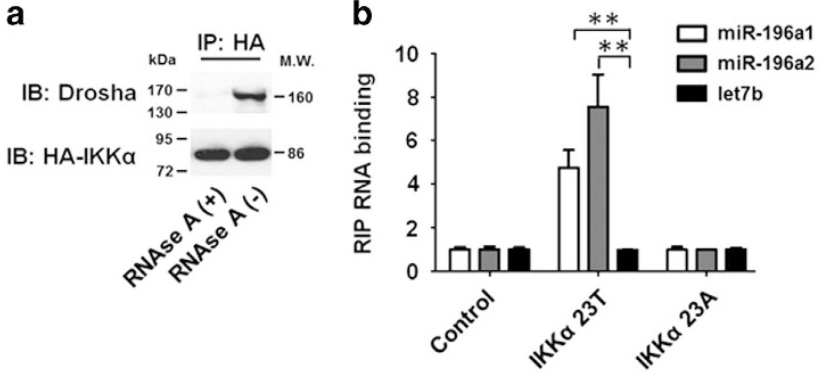

C

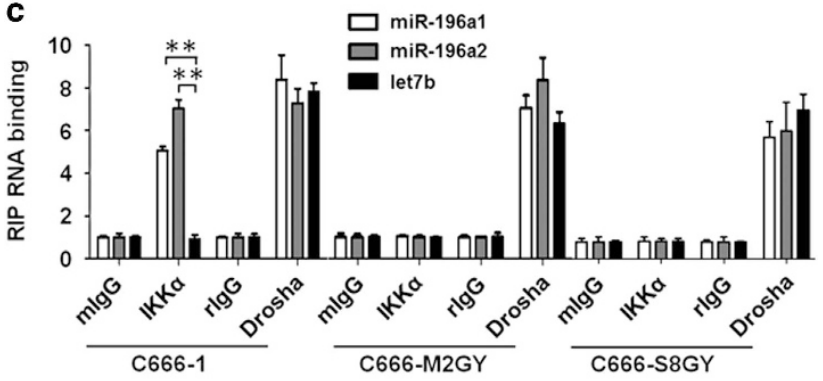

d

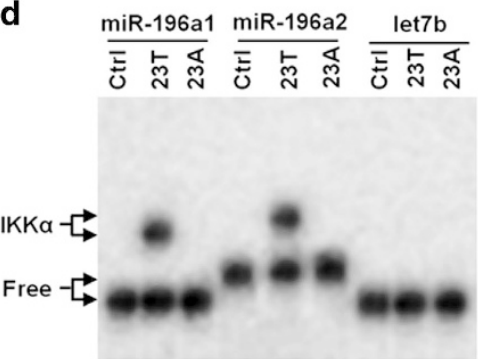

Figure 4 IKK $\alpha$ T23 phosphorylation status determines its association with primiR196a. (a) RNA dependence of interactions of IKK $\alpha$ with Drosha. Immunoprecipitates were treated with RNase A and subjected to immunoblot analysis. (b) RIP analysis of the association between IKK $\alpha$ and pri-let7b, pri-miR-196a1, primiR-196a2 in C666-IKK $\alpha-23 \mathrm{~A}$ and C666-IKK $\alpha$-23T cells. Cells were immunoprecipitated with the anti-HA, and subjected to qRT-PCR analysis with pri-let7b, miR-196a1 and miR-196a2 primers ( $\left.{ }^{\star *} P<0.01 ; n=3\right)$. (c) RIP analysis of the association between IKK $\alpha$ and pri-let7b, pri-miR-196a1, pri-miR-196a2 in C666-S8GY, C666M2GY and control C666-1 cells. Cells were immunoprecipitated with the anti-IKK $\alpha$ or anti-Drosha, and subjected to qRT-PCR analysis with pri-let7b, miR-196a1 and miR-196a2 primers. As a control, an RNA sample was immunoprecipitated with nonspecific mouse lgG (mlgG) or rabbit $\lg G$ ( $r l g G$ ) and subjected to qPCR $\left({ }^{* \star} P<0.01 ; n=3\right)$. (d) EMSA was performed with FITC-labeled pri-let7b, primiR-196a1 and pri-miR-196a2 probes and in vitro translated IKK $\alpha 23$ A and IKK $\alpha 23$ T proteins. Shifted bands as a result of specific binding to IKK $\alpha$ are shown. 'Free' indicates an unbound probe. The experiment was performed three times and a representative blot is shown

molecules (Figure 4a). To examine whether IKKa engages with pri-miR-196a in vivo, RNA immunoprecipitation (RIP) analysis was performed in both C666-IKKa-23T and C666-IKKa-23A cells. We found that HA-IKKa pulled down pri-miR-196a1 and miR-196a2, but not pri-let7b in C666-IKKa-23T cells, while HA-IKKa did not precipitate pri-miR-196a1 and miR-196a2, and pri-let7b in C666-IKKa$23 \mathrm{~A}$ cells. These results suggest that the phosphorylation status of IKKa23 $\mathrm{T}$ is important for the association of IKKa with pri-miR-196a1 and pri-miR-196a2 (Figure 4b). Consistently, anti-IKKa antibody could immunoprecipitate pri-miR-196a1 and pri-miR-196a2 in C666-1 cells, whereas it could not co-immunoprecipitate these primary miRNAs in both C666-S8GY and C666-M2GY NPC cell lines, in which IKKaT23 was dephosphorylated (Figure 4c).

To further confirm the interaction of IKKa with pri-miR-196a, an electrophoretic mobility shift assay (EMSA) was performed using in vitro translated functional full-length IKKa protein and FITC-labeled pri-miR-196a1, pri-miR-196a2 and pri-let7b as probes. Consistent with the results of the RIP assay, bands for pri-miR-196a1 and pri-miR-196a2, but not pri-let7b were shifted with the IKKa-23T but not IKKa-23A protein, and bands for pri-let7b were not shifted with either IKKa-23T or IKKa-23A protein (Figure 4d), suggesting that the specific binding of IKKa to pri-miR-196a1 and pri-miR-196a2 is dependent on the status of IKKaT23. Together, these results indicate that the specific binding of p-IKKaT23 to pri-miR-196a leads to explicit participation of $\mathrm{p}$-IKKaT23 in the biogenesis of miR-196a.

IKKa phosphorylates Drosha. To investigate whether IKKa affects the function of Drosha, we compared the expression and localization of Drosha between IKKa knockdown and control NPC cells. As Drosha is a nuclear protein, C666-1 cells had a strong nuclear Drosha staining; however, in addition to an intense nuclear Drosha staining IKKa knockdown cells also showed a diffused cytoplasmic staining of Drosha (Figures $5 \mathrm{a}$ and b). We also performed immunofluorescence of Drosha in C666-IKKa-23T and C666-IKKa23A cells. Interestingly, C666-IKKa-23T cells had a unique and strong nuclear Drosha staining while C666-IKKa-23A cells showed both nuclear and cytoplasmic staining of Drosha, suggesting that the phosphorylation status of IKKaT23 is associated with the localization of Drosha (Figure 5c). Supporting this phenomenon, Drosha was uniquely stained in nucleus of control C666-1 cells while it was stained in both nucleus and cytoplasm of radioresistant C666-S8GY and C666-M2GY NPC cells, in which IKKa was dephosphorylated at T23 site (Figure $5 d$ ).

It has been reported that the phosphorylation of Drosha is essential for it nuclear localization. ${ }^{21}$ The diffuse staining of Drosha in the cytoplasm of IKKa knockdown cells suggests that IKKa may phosphorylate Drosha. To verify this hypothesis we performed an in vitro kinase assay using an active IKKa protein and a recombinant Drosha protein, and we found that IKKa phosphorylated Drosha in vitro (Figure 5e). Together, these results suggest that phosphorylation of IKKa at $23 \mathrm{~T}$ site promotes the association of IKKa with a group of pri-miRNAs, including miR-196a, where IKKa phosphorylates Drosha, leading to increased processing of these miRNAs (Figure 5f).

IKKaT23-mediated miR-196a biogenesis regulates the sensitivity of NPC cells to radiation. As miR-196a was downregulated in radioresistant NPC cells, its expression may be related to the sensitivity of NPC cells to radiation treatment. To test this hypothesis, both C666-S8GY and C666-M2GY cells were incubated with miR-196a mimics, followed by treatment with radiation. We found that miR-196a mimics restored the sensitivity of C666-S8GY and C666M2GY to radiation treatment (Figure 6a), suggesting that miR-196a is a key regulator of NPC cells' response to radiation treatment. 
a
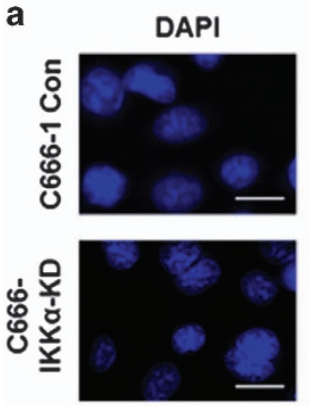

c
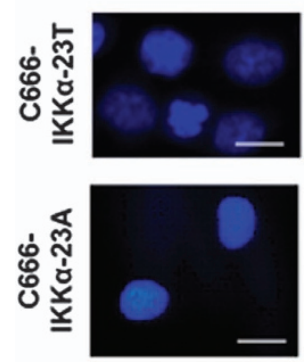

e

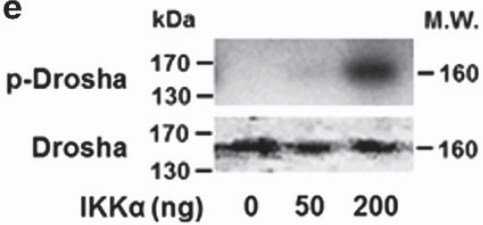

M.W. b
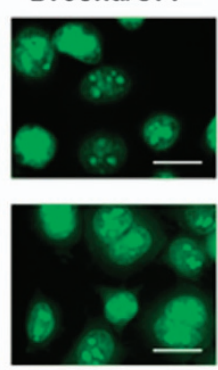

Drosha/GFP
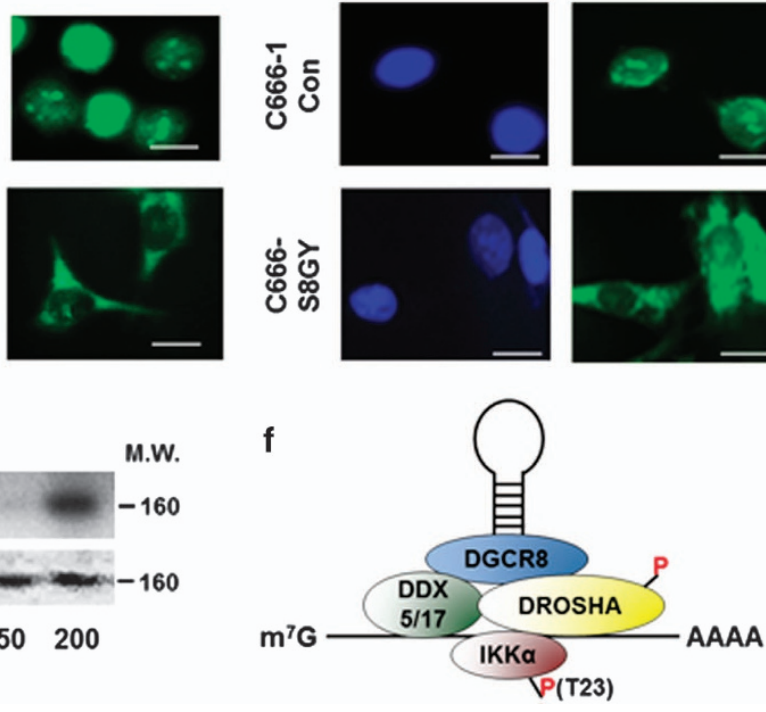

Figure 5 IKK $\alpha$ phosphorylates Drosha. (a) Endogenous Drosha staining in C666-IKK $\alpha$-KD and control C666-1 NPC cells ( $\times 400)$. Scale bars represent $50 \mu \mathrm{m}$. (b) Endogenous Drosha staining in IKK $\alpha$ knockdown and control Myc-CaP cells ( $\times 400)$. Scale bars represent $50 \mu \mathrm{m}$. (c) Endogenous Drosha staining in C666-IKK $\alpha-23 \mathrm{~A}$ and C666-IKK $\alpha-23 \mathrm{~T}$ cells $(\times 400)$. Scale bars represent $50 \mu \mathrm{m}$. (d) Endogenous Drosha staining in C666-S8GY cells and control C666-1 cells ( $\times 400)$. Scale bars represent $50 \mu \mathrm{m}$. (e) In vitro phosphorylation of Drosha by an active IKK $\alpha$ protein in the presence of $5 \mu \mathrm{Ci}\left[\gamma^{32} \mathrm{P}\right]-A T P$ and $200 \mu \mathrm{M}$ cold ATP. The reaction was resolved on SDS-PAGE, and the phosphorylation of Drosha was detected by autoradiography. Coomassie blue staining of the SDS-PAGE gel was also shown for loading control. (f) IKK $\alpha$ regulates miRNA biogenesis. IKK $\alpha$ recognizes pri-miRNA, associates with the Drosha microprocessor, phosphorylates Drosha and enhances miRNA processing

To further investigate the role of miR-196a in p-IKKa23 T-mediated regulation of NPC cells' response to radiation treatment, C666-IKKa-23T cells that had high levels of endogenous miR-196a were incubated with miR-196a inhibitors or vehicle while C666-IKKa-23A cells that had a low level of endogenous miR-196a were incubated with miR-196a mimics or vehicle. After $12 \mathrm{~h}$, these cells were treated by radiation and subjected to MTT, soft agar colony formation and tumor sphere formation assays. We found that C666-IKKa23T cells incubated with miR-196a inhibitors were significantly more resistant to radiation treatment (Figure 6b), and formed more colonies in soft agar (Figure $6 \mathrm{c}$ ) and more tumor spheres in suspension culture (Figure 6d) than the cells incubated with vehicle. In contrast, C666-IKKa-23A cells incubated with miR-196a mimics were considerably more sensitive to radiation treatment, and had less cell viability (Figure 6e) and less colonies in soft agar (Figure 6f), and formed less and smaller tumor spheres in suspension culture (Figure $6 \mathrm{~g}$ ) than the cells incubated with vehicle. These results suggest that overexpression of miR-196a sensitizes NPC cells to radiation treatment while downregulation of miR-196a protects cells from radiation-induced cell death.

IKKaT23-mediated regulation of miR-196a is associated with the radioresistance of clinical human NPC. To investigate the relevance of IKKaT23-mediated regulation of miR-196a to radiotherapy resistance of clinical human NPC, we examined the expression of p-IKKaT23 and miR-196a by immunohistochemistry and in situ hybridization, respectively, in paraffin-embedded sections of 116 cases of NPC. Fortyseven cases of the NPC were radiotherapy resistance while 69 cases were radiotherapy sensitive. We found that most of radiosensitive NPC cases had strong p-IKKaT23 staining while most of radioresistant NPC cases had weak or moderate p-IKKaT23 expression. The expression levels of $\mathrm{p}-\mathrm{IKKaT23}$ in radiosensitive and radioresistant NPC tissues were significantly different (Figures $7 a$ and b). Similarly, miR-196a was strongly expressed in most of the radiosensitive NPC tissues while the expression of miR-196a in most of radioresistant NPC tissues was weak or moderate. The expression levels of $\mathrm{miR}-196 \mathrm{a}$ in radiosensitive and 


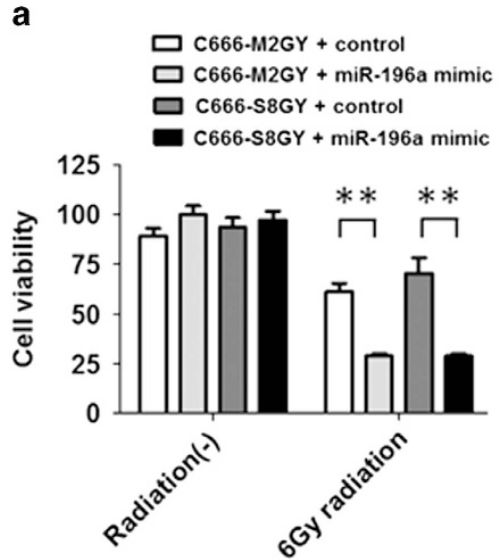

c
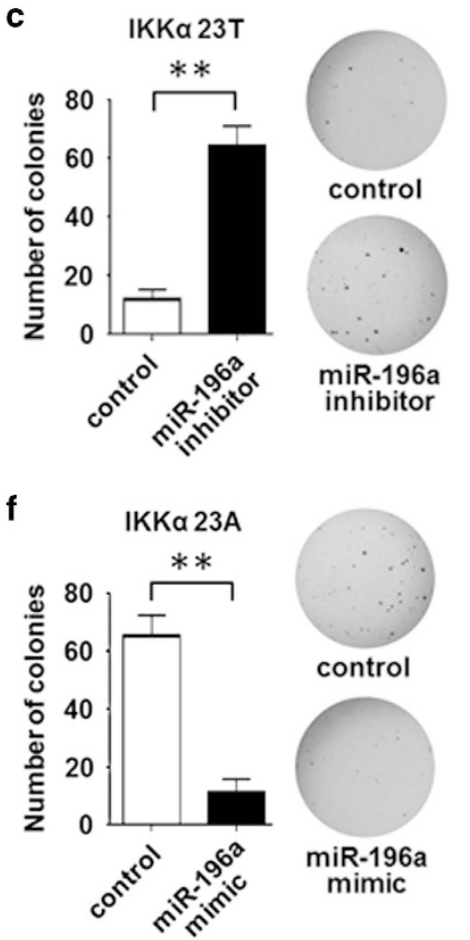

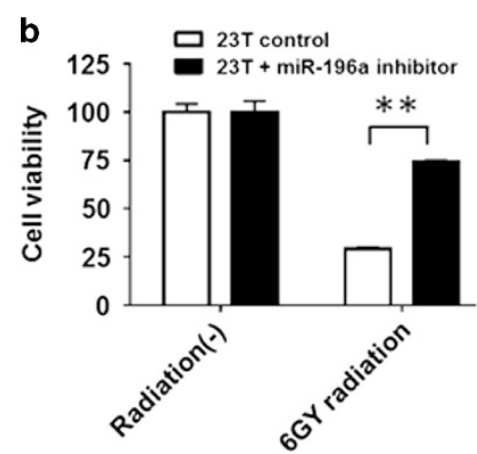

d
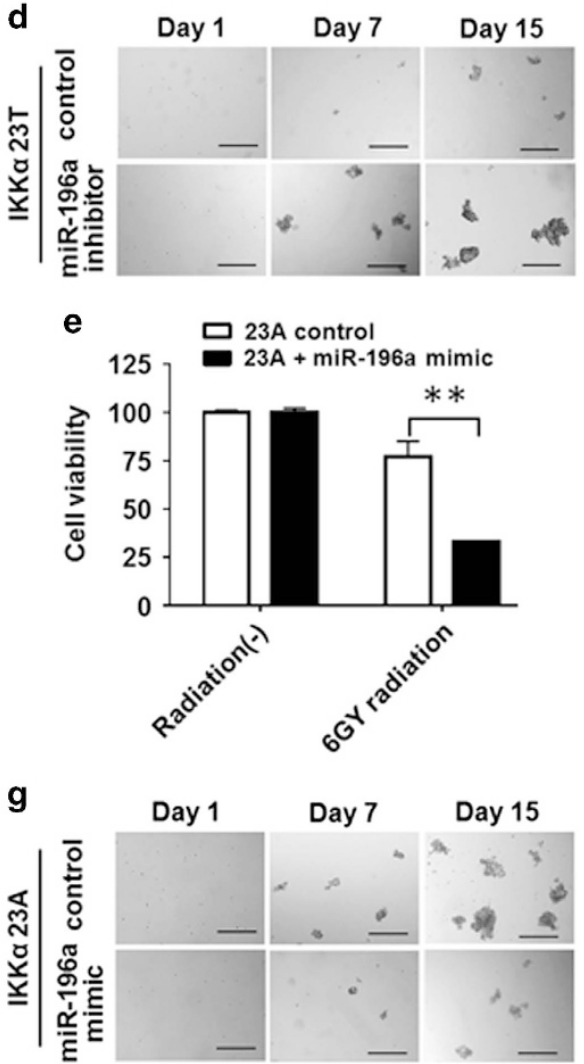

Figure 6 IKK $\alpha$ T23-mediated regulation on pri-miR-196a processing plays important roles in NPC radioresistance. (a) MTT assay shows cell viability of C666-S8GY and C666-M2GY cells incubated with miR-196a mimics, followed by 6 Gy of radiation treatment. (b) MTT assay shows cell viability of C666-IKK $\alpha$-23T cells incubated with miR-196a inhibitors, followed by 6 Gy of radiation treatment. (c) Soft agar colony formation of C666-IKK $\alpha$-23T cells incubated with miR-196a inhibitors, followed by 6 Gy of radiation treatment. (d) Tumor spheres formation of C666-IKK $\alpha$-23T cells incubated with miR-196a inhibitors, followed by 6 Gy of radiation treatment. Scale bars represent $100 \mu \mathrm{m}$. (e) MTT assay shows cell viability of C666-IKK $\alpha$-23A cells incubated with miR-196a mimics, followed by 6 Gy of radiation treatment. (f) Soft agar colony formation of C666-IKK $\alpha-23 \mathrm{~A}$ cells incubated with miR-196a mimics, followed by 6 Gy of radiation treatment. (g) Tumor spheres formation of C666-IKK $\alpha$-23A cells incubated with miR-196a mimics, followed by 6 Gy of radiation treatment. Scale bars represent $100 \mu \mathrm{m}$. Note: ${ }^{* *} P<0.01$

radioresistant NPC tissues were significantly different (Figures 7c and d). Furthermore, the expression of p-IKKaT23 was positively correlated with the expression of miR-196a in human NPC tissues (Figure 7e).

\section{Discussion}

Radiotherapy is the most commonly applied treatment for undifferentiated NPC, but tumor recurrence is essentially universal due to marked radioresistance. ${ }^{2,3}$ Ionizing radiation (IR) causes damage to cellular structures of irradiated cells, including DNA. IR induces DNA damage either by directly damaging the DNA or by indirectly generating reactive oxygen species, which ultimately alter the chemical structure of DNA, leading tumor cells to halting cellular proliferation and initiating a DNA damage response. With extensive DNA damage, a cell usually undergoes cell death, which is the mechanism by which radiation functions therapeutically to reduce tumor burden. However, tumor cells often possess or acquire radioprotective capabilities that enable them to escape radiation-induced cell death. Several signaling pathways, including $\mathrm{PI} 3 \mathrm{~K} / \mathrm{AKT}$, mitogen-activated protein kinase and 

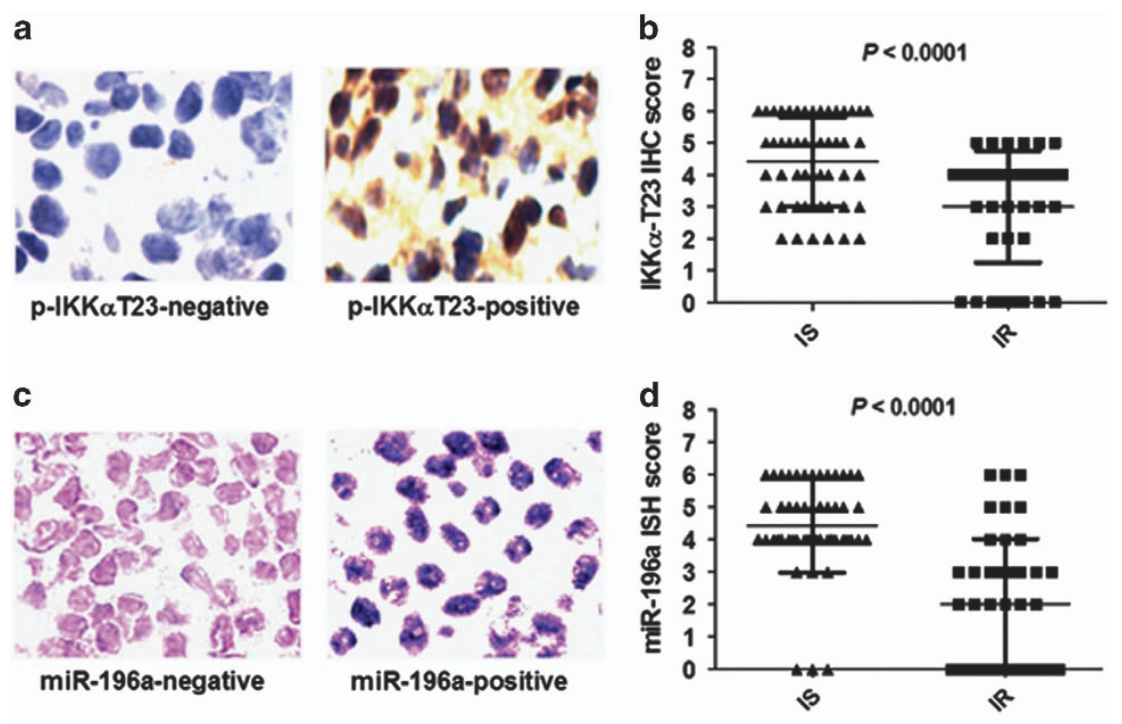

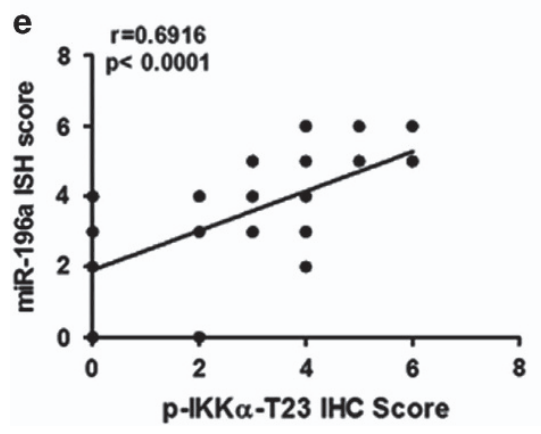

Figure 7 IKK $\alpha$ T23-mediated regulation of miR-196a is related to radioresistance of human NPC. (a) IHC of p-IKK $\alpha \mathrm{T} 23$ ( $\times 400)$. (b) Correlation between p-IKK $\alpha \mathrm{T} 23$ expression and human NPC radioresistance. (c) ISH of miR-196a (×400). (d) Correlation between miR-196a expression and human NPC radioresistance. (e) Correlation between $\mathrm{p}-\mathrm{IKK} \alpha \mathrm{T} 23$ and miR-196a expression in human NPC tissues

ATM/CHK2/p53, have been linked to the tumor radioresistance. ${ }^{22,23}$ In the present study we have demonstrated that IKKa-mediated miR-196a biogenesis regulates the sensitivity of NPC cells to radiotherapy. Dephosphorylation of p-IKKaT23 downregulates miR-196a expression and promotes the resistance of NPC cells to radiation treatment.

The biogenesis of mature miRNAs is a multistep process that starts with the initial transcription of their genes by RNA polymerase II, resulting in long, capped and polyadenylated primary miRNAs (pri-miRNA). The pri-miRNA are processed in the nucleus by the Drosha complex, releasing a $\sim 65-70$ nucleotide hairpin structure precursor miRNA (pre-miRNA). The pre-miRNA is then exported to the cytoplasm and cleaved by Dicer into double stranded miRNAs. ${ }^{24-26}$

It has been reported that Drosha associates with dozens of distinct polypeptides, including DGCR8, to form a large 'microprocessor' Drosha complex. ${ }^{27}$ The processing of primiRNA by the Drosha complex takes place concurrently with or shortly after transcription. ${ }^{28}$ DGCR8, which can directly interact with pri-miRNA, assists this process by correctly positioning Drosha on the pri-miRNA. ${ }^{29}$ Other proteins, including Lin28, nuclear ribonucleoprotein (hnRNP) A1, R-Smads, p53 and BRCA1, interact with Drosha and specifically regulate the processing of a group of pri-miRNA. Lin28 and hnRNP A1 interact with Drosha by binding to the terminal loop region of pri-let-7 and pri-miR-18a, respectively. ${ }^{30,31}$ The signal transducers of the transforming growth factor $\beta /$ bone morphogenetic protein, the Smads, promote the expression of a subset of miRNAs, including miR-21 and miR-199a, by facilitating the cleavage reaction by Drosha. ${ }^{16,32}$ In response to DNA damage, p53 interacts with the Drosha processing complex through its association with DEAD-box RNA helicase p68 (also known as DDX5) and facilitates the processing of primary miRNAs to precursor miRNAs, which enhances the post-transcriptional maturation of several miRNAs with growth-suppressive function, including miR-16-1, miR-143 and miR-145. ${ }^{18}$ Tumor suppressor BRCA1 regulates the biogenesis of a group of miRNA biogenesis, including let-7a-1, miR-16-1, miR-145 and miR-34a, via direct association with the Drosha microprocessor complex and Smad3/p53/DHX9. ${ }^{17}$

In this study, we identified a set of miRNAs that is regulated post-transcriptionally by IKKa. IKKa is a component of the IKK complex. ${ }^{5}$ In addition to its cytoplasmic functions where it controls the activation of both classical and alternative NF- $\kappa$ B pathways, ${ }^{6,9-12}$ IKKa can function as a nuclear protein kinase ${ }^{33-35}$ and nuclear IKKa plays important roles in prostate cancer progression and metastasis. ${ }^{13,14}$ In the present study we have shown that IKKa controls the biogenesis of a group of miRNA, including miR-196a. Phosphorylation of IKKa at 23 T site promotes the association of IKKa with pri-miRNAs, where 
IKKa phosphorylates Drosha, leading to increased biogenesis of these miRNAs.

MiR-196a has two forms: miR-196a1 and miR-196a2. Although miR-196a1 and miR-196a2 are transcribed from two different genes, the mature nucleotide sequences of miR-196a1 (Promega, Madison, WI, USA) and miR-196a2 are identical. It has been reported that miR-196a plays important role in development, immunity, cancer and cell differentiation. ${ }^{36}$ However, the detailed functions and molecular mechanisms by which miR-196a contributes to these physiological and pathological process remain largely unknown. ${ }^{36}$ Particularly, the roles of miR-196a in cancer are elusive. Some reports suggest that miR-196a plays an oncogenic role in tumor development, ${ }^{37,38}$ while others indicate that miR-196a may be a tumor suppressor. For instance, it was reported that the expression of miR-196a was dramatically decreased in melanoma cells when compared with healthy control melanocytes, and the low miR-196a expression in melanoma cells enhanced migratory potential of these melanoma cells. ${ }^{39}$ Moreover, enforced expression of miR-196a reduced in vitro invasion and in vivo spontaneous metastasis of breast cancer cells. ${ }^{40}$ In addition, overexpression of miR-196a inhibited the proliferation of human adipose tissue-derived mesenchymal stem cells while enhancing osteogenic differentiation. ${ }^{41}$ Our results show that NPC cells become radioresistant by reducing the expression of miR-196a, and expression of miR-196a restores the sensitivity of radioresistant NPC to radiation therapy. Importantly, the expression level of miR-196a in clinical NPC samples is correlated with the sensitivity of NPC to radiotherapy. Our study suggests that miR-196a is a good clinical biomarker for predicting the sensitivity of NPC to radiotherapy and miR-196a is a potential radiosensitizer for NPC radiotherapy.

\begin{abstract}
Materials and Methods
Cell cultures. The human NPC cell lines C666-1, ${ }^{42} \mathrm{HONE}^{43}$ and the mouse prostate cancer cell line Myc-CaP were cultured in RPMI-1640 medium (Lonza, Walkersville, MD, USA) supplement with $10 \%$ fetal bovine serum (Atlanta Biological, Flowery Branch, GA, USA) and $1 \%$ penicillin-streptomycin solution (Thermo scientific, Waltham, MA, USA). The immortalized normal nasopharyngeal epithelial cell line NP69 ${ }^{44}$ were cultured and grown in defined keratinocyte-SFM (Life Technologies, Carlsbad, CA, USA) with growth supplement and bovine pituitary extract (Sigma-Aldrich, St. Louis, MO, USA) and penicillin-streptomycin. Cells were cultured at $37^{\circ} \mathrm{C}$ with $5 \% \mathrm{CO}_{2}$ in an incubator.
\end{abstract}

Antibodies. The following antibodies were used: IKK $\alpha$ antibody (Novus Biological, Littleton, CO, USA; \#NBP2-27409), phospho-IKK $\alpha$ antibody (Thr23; Sigma-Aldrich; \#SAB4300106), phospho-IKK $\alpha$ antibody (Ser176/180; Cell Signaling; \#2697S), Drosha antibody (Cell Signaling; \#3364S), HA antibody (Roche, Indianapolis, IN, USA; \#11867423001), normal mouse IgG (Santa Cruz; \#sc-2025), normal rabbit IgG (Santa Cruz; \#sc-2027), $\beta$-actin antibody (Santa Cruz; \#sc-47778), HRP-linked anti-mouse IgG (GenScript, Piscataway, NJ, USA; \#A00160), HRP-linked anti-rabbit IgG (Santa Cruz; \#sc-2004), FITC conjugated anti-mouse IgG (Sigma).

Cell irradiation and the establishment of radioresistant NPC cells. Two approaches were employed to establish radioresistant NPC cell lines. Approach 1: NPC C666-1 and HONE1 cells were seeded at a density of $1.5 \times 10^{6}$ per well in a six-well plate and cultured overnight. Cells were irradiated at the dosage of 2 Gy per day for consecutive five days per week for 4 weeks to establish radioresistant NPC cells, named as C666-1-M2GY and NONE1-M2GY. Approach 2 : C666-1 and HONE1 cells at a density of $2 \times 10^{5}$ per well in a 24-well plate were given a sublethal does of $8 \mathrm{~Gy}$, and $48 \mathrm{~h}$ later cells were dissociated into single cells and seeded in $15 \mathrm{~cm}$ dish. Three weeks later large colonies were picked and examined for the radiation resistance. These colonies were named C666-1-S8GY and HONE1-S8GY. MTT, soft agar colony formation and tumor sphere formation assay were performed to assess the radioresistance of these cells.

MTT assay. Cell viability was measured by the 3-(4,5-dimethylthiazol-2-yl)-2,5diphenyltetrazoliumbromide (MTT) (Life Technologies) colorimetric assay. Briefly cells were seeded in 96-well plates at a density of $10 x^{3}$ cells per well, after 6 days incubation, MTT solution (Life Technologies) was added to wells and incubated for $4 \mathrm{~h}$ in $37^{\circ} \mathrm{C}$. The absorbance was measured at $570 / 630 \mathrm{~nm}$ using a spectrophotometer (SpectraMax M5; Molecular Devices Corp, Sunnyvale, CA, USA).

Soft agar colony formation assay. To assess anchorage independent growth, 2000 cells were seeded in medium containing $0.35 \%$ agarose (LowMelt Agarose; GeneMate, Kaysville, UT, USA) on top of $0.7 \%$ agarose/media. After solidifying the cells were covered with media. Media was changed two times every week. Colonies were stained with $0.01 \%$ crystal violet (Alfa Aesar, Ward Hill, MA, USA) and examined after 25 days of culture. Colonies larger than $50 \mu \mathrm{M}$ were enumerated.

Tumor sphere formation assay. For tumor sphere formation assay, cells were dissociated into single cells and seeded in low cell density $\left(5 \times 10^{2}\right)$ on a sixwell ultra-low cluster plate (Sigma-Aldrich), and cultured with serum-free DMEM/F12 (Invitrogen, Carlsbad, CA, USA), $20 \mathrm{ng} / \mathrm{ml}$ EGF (Sigma-Aldrich), $20 \mathrm{ng} / \mathrm{ml}$ bFGF (Cell Signaling, Beverly, MA, USA) and $20 \mathrm{ng} / \mathrm{ml}$ insulin (Cell Signaling). The images of tumor spheres were captured under a microscope equipped with a camera at days 1,7 and 15 of culture.

Western blot analysis. Cells were collected and lysed with ice-cold lysis buffer containing $0.25 \%$ protease inhibitor (Calbiochem, La Jolla, CA, USA) and phosphatase inhibitor (Roche). Samples were resolved on $8-15 \%$ SDS-polyacrylamide gel and transferred to PVDF membrane (Millipore, Billerica, MA, USA). The membrane was blocked with milk or $5 \%$ BSA (Fisher Scientific, Hanover Park, IL, USA), and incubated with primary antibodies, followed by incubation with corresponding secondary antibodies. An ECL western blotting substrate (Thermo Scientific) was added and chemiluminescence signal was detected.

Immunoprecipitation and immunoblot assays. For C666-1, HONE1 and Myc-CaP cells, $500 \mu \mathrm{g} / 400 \mu \mathrm{l}$ cleared lysates were incubated with the antiIKK $\alpha$ /DROSHA or control IgG antibodies, Protein A/G PLUS-Agarose Beads (Santa Cruz, Dallas, TX, USA) overnight at $4{ }^{\circ} \mathrm{C}$. Beads were washed three times with stringent buffer, and then mixed with $20 \mu$ l of loading buffer and incubated at $95^{\circ} \mathrm{C}$ for $5 \mathrm{~min}$. After centrifugation, supernatants were subjected to SDS-PAGE and transferred to PVDF membrane. Immunoblotting was performed with the indicated antibodies. For C666-IKK $\alpha-23$ T and C666-IKK $\alpha-23$ A stable cell lines, Pierce Anti-HA Magnetic Beads (Thermo Scientific) were used according to the manufacturer's instructions.

qRT-PCR assays. qRT-PCR assays were performed to determine the expression levels of primary, precursor and mature miRNAs. Briefly, total RNA was purified using RNeasy Mini Kit (Qiagen, Valencia, CA, USA), and then reverse transcribed with High-Capacity Reverse Transcription Kit (Applied Biosystems by Life Technologies). Mature miRNA was prepare using mirVana miRNA Isolation Kit (Ambion by Life Technologies), and then reverse transcription was carried out using Universal CDNA Synthesis Kit II (Exiqon, Woburn, MA, USA). The qPCR reaction was performed using a $5 \times$ Hot FIREPol EvaGreen qPCR SuperMix (Solis BioDyne, Tartu, Estonia), according to the manufacturer's instructions. Data analysis was performed using the comparative $\mathrm{Ct}$ method. Results were normalized to human 18S rRNA for pri- and pre-miRNAs, or human 5S rRNA for mature miRNAs. The primer sequences used are shown in Supplementary Table S1.

Xenograft mouse model. Six-week-old Rag $1^{-1-}$ mice were subcutaneously inoculated with $1 \times 10^{7} \mathrm{NPC}$ cells with Matrigel Matrix (Corning, Tewksbury, MA, USA). Tumor formation was measured every 3 days for 30 days. The tumor volumes were calculated by the equation $V\left(\mathrm{~mm}^{3}\right)=a \times b^{2} / 2$, where ' $a$ ' is the largest diameter and ' $b$ ' is the perpendicular diameter.

Transfections. We transfected cells with hsa-miR-196a Mimic (HMI0322; Sigma-Aldrich), hsa-miR-196a inhibitor (HSTUD0322; Sigma-Aldrich) and different kinds of plasmids using the TransIT-LT1 transfection reagent (Mirus, Madison, WI, USA), 
according to manufacturer's recommendation. C666-IKK $\alpha-23 \mathrm{~T}$ and $\mathrm{C} 666-\mathrm{IKK} \alpha-23 \mathrm{~A}$ stable cell lines were achieved by geneticin (Teknova, Hollister, CA, USA) selection. All transfection experiments were done in triplicates and repeated for three times.

shRNA lentivirus infection. C666-1 cells were infected with lentivirus expressing IKK $\alpha$ shRNA (Sigma-Aldrich) and empty vector with the LTV-201 (Cell Biolabs, Inc, San Diego, CA, USA). Cells were selected by puromycin. The efficiency of IKK $\alpha$ knockdown was determined by western blot.

In vivo monitoring of pri-miRNA processing. Plasmid constructs with pri-miRNA (let7b, miR-196a1, miR-196a2) at the $3^{\prime}$ untranslated region of firefly luciferase $\mathrm{CDNA}$ and expression vectors were transfected into C666-1 cells. Cell extracts were prepared at $48 \mathrm{~h}$ after transfection, and the ratio of firefly and renilla luciferase was measured using a Dual-Luciferase Reporter Assay system (Promega). Values were further normalized by that of an empty vector and are indicated with standard deviation. The primer sequences used for cloning are shown in Supplementary Table S1.

In vitro pri-miRNA processing analysis. Purified PCR products containing a T7 sequence and a $125-160$ bp of pri-miR-196a1, pri-miR-196a2 and pri-let7b were transcribed in vitro (TranscriptAid T7 High Yield Transcription Kit; Thermo Scientific) and internally labeled with FITC (Fluorescein RNA Labeling Mix; Roche). Pri-miRNA was denatured for $5 \mathrm{~min}$ by heating at $65^{\circ} \mathrm{C}$ and re-natured by gradually decreasing the temperature. At the same time, immunoprecipitated Drosha, immunoprecipitated IKK $\alpha$ complex from C666-1, immunoprecipitated HAIKK $\alpha 23 \mathrm{~T}$ and immunoprecipitated IKK $\alpha 23 \mathrm{~A}$ complex from respective stable cell lines were prepared. Each processing reaction contained one of above-mentioned immunoprecipitated protein, FITC-labeled pri-miRNA, $\mathrm{MgCl}_{2}$, ATP and RNase inhibitor. The reaction mixture was incubated at $37^{\circ} \mathrm{C}$ for $120 \mathrm{~min}$. After purification (RNeasy Mini Kit; Qiagen), RNA was loaded on $8 \%$ urea denaturing polyacrylamide gels, then analyzed using a E-Gel Imager (Life Technologies). The primer sequences used for PCR are shown in Supplementary Table S1.

RIP. All buffers used in RIP contained $0.5 \mathrm{U} / \mu$ l RNase inhibitor (Applied Biosystems, Carlsbad, CA, USA). First, nuclei were isolated from $0.75 \%$ formaldehyde-fixed cells and chromatin was sheared using a homogenizer. After immunoprecipitation with anti-IKK $\alpha$ or anti-DROSHA or anti-HA antibodies, the precipitated RNA was isolated by using TRIzol RNA extraction reagent (Life Technologies). An aliquot of RNA was used for a cDNA synthesis reaction and GPCR analysis. The primer sequences used are shown in Supplementary Table S1.

EMSA. FITC-labeled pri-miR-196a1, pri-miR-196a2 and pri-let7b were prepared by using an in vitro transcription system as mentioned above. Recombinant IKK $\alpha 23$ T and IKK $\alpha 23$ A protein were synthesized by 1-Step Human High Yield In Vitro Translation Kits (Thermo Scientific). Denatured and re-natured pri-miRNA was mixed with recombinant protein at $4{ }^{\circ} \mathrm{C}$ for $1 \mathrm{~h}$ in EMSA buffer (10 mM Tris-HCl, pH 7.5, $150 \mathrm{mM} \mathrm{KCl,} \mathrm{10 \%} \mathrm{glycerol,} 50 \mu \mathrm{g} / \mathrm{ml}$ poly[dl $-\mathrm{dC}$ ] and RNase inhibitor). Bound complexes were resolved on $6 \%$ native polyacrylamide gels in $0.5 \times$ Tris/borate/EDTA buffer and then analyzed using a E-Gel Imager (Life Technologies).

Tissues. Two hundred eighty-six NPC patients who were treated by curativeintent radiotherapy (a total dose of 60-70 Gy) using a modified linear accelerator in the Xiangya Hospital of Central South University, China from February 2004 to July 2013 were reviewed. All these NPC patients were followed-up after the first radiotherapy. Among these patients, $116 \mathrm{NPC}$ patients without distant metastasis (MO; WHO staging II and III) at the time of diagnosis, comprising 47 radioresistant and 69 radiosensitive patients, were recruited in this study. Radioresistant NPC patients were defined as ones with persistent disease (incomplete regression of tumor) at $>6$ weeks after completion of radiotherapy or ones with recurrent disease at the nasopharynx and/or neck nodes at $>2$ months after completion of radiotherapy. ${ }^{45,46}$ Radiosensitive NPC patients were defined as ones without the local residual lesions or recurrence 5 years after completion of radiotherapy. Diagnoses were established by experienced pathologists and NPC oncologist based on the 1978 WHO classification. The study was approved by the ethics committee of Xiangya School of Medicine, Central South University, China.
Immunohistochemistry. The expression of $\mathrm{p}-\mathrm{IKK} \alpha \mathrm{T} 23$ in clinic NPC was performed on paraffin-embedded primary NPC tissue sections by immunohistochemical analysis. Briefly, 4- $\mu$ m-thick tissue sections were treated with an antigen retrieval solution (10 mmol// sodium citrate buffer $(\mathrm{pH} 6.0))$, incubated (overnight, $4{ }^{\circ} \mathrm{C}$ ) with anti-p-IKK $\alpha \mathrm{T} 23$ antibody ( $1: 300$ dilution), then incubated with biotinylated secondary antibody (1:500 dilution), followed by avidin-biotin peroxidase complex (Dako, Carpinteria, CA, USA). Finally, tissue sections were stained with $3^{\prime}, 3^{\prime}$ diaminoben-zidine (Sigma) and counterstained with Harris' modified hematoxylin. In negative controls, primary antibodies were omitted.

All sections were examined and assessed independently by two investigators. A quantitative score was performed by adding the score of staining area and the score of staining intensity for each case to assess the protein expression levels as previously described. ${ }^{47}$ At least 10 high-power fields were chosen randomly and $>1000$ cells were counted for each section. First, a quantitative score was performed by estimating the percentage of staining-positive cells: 0 , no staining of cells in any microscopic field; $1+,<30 \%$ of cells stained positive; $2+$, between 30 and $60 \%$ stained positive; and $3+,>60 \%$ stained positive. Second, the staining intensity score was evaluated as: 0 , no staining; $1+$, mild staining; $2+$, moderate staining; $3+$, intense staining. Finally a total score (ranging from 0 to 6 ) was obtained by adding the area score and the intensity score for each case. A combined staining score of $\leqslant 2$ was considered to be negative staining (no expression); a score between 3 and 4 was considered to be moderate staining (expression); and a score between 5 and 6 was considered to be strong staining (high expression).

In situ hybridization. In situ hybridization was performed according to the manufacturer's protocol for formalin-fixed, paraffin-embedded tissue sections. The probes were $3^{\prime}, 5^{\prime}$-labeled with digoxigenin with the DIG tailing kit (Exiqon). After deparaffinization, the NPC tissue sections were subjected to proteinase $\mathrm{K}(40 \mu \mathrm{g} /$ $\mathrm{ml}$ ) digestion $(10 \mathrm{~min})$. The prehybridization $(1 \mathrm{~h})$ and hybridization $(2 \mathrm{~h})$ were subsequently treated by the manufacturer's instruction of the locked nucleic acidmodified probes. For the immuno detection, tissues were incubated overnight at $4^{\circ}$ $C$ in anti-DIG-AP FAB fragment $(1: 1000)$. The final visualization was carried out with BCIP/NBTP. The stained sections were reviewed and scored under a light microscope (LEI CA DM5000 B, Buffalo Grove, IL, USA) independently by two investigators. The staining intensity score was evaluated as: $1=$ no staining or weak staining, 2 = intermediate staining, $3=$ strong staining; and the percentage of tissue cell staining was scored as: $0=<10 \%, 1=10-30 \%, 2=10-30 \%, 3=>60 \%$ ). A combined staining score of $\leqslant 2$ was considered to be negative staining (no expression); a score between 3 and 4 was considered to be moderate staining (expression); and a score between 5 and 6 was considered to be strong staining (high expression).

Statistical analysis. Statistical analyses were performed using the statistical package SPSS 13.0 and Graphpad Prism 5. Difference of the protein expression between radiosensitive and radioresistant NPC tissues was analyzed using Student's $t$-test. The expression of p-IKK $\alpha$ T23 and miRNA-196 $\alpha$ in NPC tissues was assessed for correlations. A two-sided $P<0.05$ was considered significant.

\section{Conflict of Interest}

The authors declare no conflict of interest.

Acknowledgements. We thank Dr. Xiaoli Tang and Dr. Bharat Ramratnam at Warren Alpert Medical School of Brown University for kindly providing us with GFPDrosha plasmid. This work was supported by grants from the United States Department of Defense (W81XWH-09-1-0533) and National Institute of Health (1R01CA140956, 1R21NS073098) to J-LL, and China Nature Science Foundation (81472847, 81372905) to J-LL and XPF, by Scholarships from China Scholarship Council (CSC) to XPF and XL, and an UICC YY fellowship (Feng2014/YY1/315514) to XPF.

1. Yu MC, Yuan JM. Epidemiology of nasopharyngeal carcinoma. Semin Cancer Biol 2002; 12 : 421-429.

2. Lee AW, Poon YF, Foo W, Law SC, Cheung FK, Chan DK et al. Retrospective analysis of 5037 patients with nasopharyngeal carcinoma treated during 1976-1985: overall survival and patterns of failure. Int J Radiat Oncol Biol Phys 1992; 23: 261-270. 
3. Hui EP, Leung SF, Au JS, Zee B, Tung S, Chua D et al. Lung metastasis alone in nasopharyngeal carcinoma: a relatively favorable prognostic group. A study by the Hong Kong Nasopharyngeal Carcinoma Study Group. Cancer 2004; 101: 300-306.

4. Wang WJ, Wu SP, Liu JB, Shi YS, Huang X, Zhang QB et al. MYC regulation of CHK1 and CHK2 promotes radioresistance in a stem cell-like population of nasopharyngeal carcinoma cells. Cancer Res 2013; 73: 1219-1231.

5. Rothwarf DM, Karin M. The NF-kappa B activation pathway: a paradigm in information transfer from membrane to nucleus. Sci STKE 1999; 1999: RE1.

6. Karin M, Ben-Neriah Y. Phosphorylation meets ubiquitination: the control of NF-[kappa]B activity. Annu Rev Immunol 2000; 18: 621-663.

7. Greten FR, Karin M. NF-kB: linking inflammation and immunity to cancer development and progression. Nat Rev Immunol 2005; 5: 749-759.

8. Luo JL, Kamata H, Karin M. IKK/NF-kappaB signaling: balancing life and death-a new approach to cancer therapy. J Clin Invest 2005; 115: 2625-2632.

9. Ghosh S, Karin M. Missing pieces in the NF-kB puzzle. Cell 2002; 109 (Suppl):S81-S96.

10. Bonizzi G, Karin M. The two NF-kB activation pathways and their role in innate and adaptive immunity. Trends Immunol 2004; 25: 280-288.

11. Senftleben U, Cao Y, Xiao G, Greten FR, Krahn G, Bonizzi G et al. Activation by IKKa of a second, evolutionary conserved, NF-kB signaling pathway. Science 2001; 293: 1495-1499.

12. Bonizzi G, Bebien M, Otero DC, Johnson-Vroom KE, Cao Y, Vu D et al. Activation of IKKa target genes depends on recognition of specific kB binding sites by RelB:p52 dimers. EMBO J 2004; 23: 4202-4210.

13. Luo JL, Tan W, Ricono JM, Korchynskyi O, Zhang M, Gonias SL et al. Nuclear cytokineactivated IKKalpha controls prostate cancer metastasis by repressing Maspin. Nature 2007; 446: 690-694.

14. Ammirante M, Luo JL, Grivennikov S, Nedospasov S, Karin M. B-cell-derived lymphotoxin promotes castration-resistant prostate cancer. Nature 2010; 464: 302-305.

15. Chen K, Rajewsky N. The evolution of gene regulation by transcription factors and microRNAs. Nat Rev Genet 2007; 8: 93-103.

16. Davis BN, Hilyard AC, Lagna G, Hata A. SMAD proteins control DROSHA-mediated microRNA maturation. Nature 2008; 454: 56-61.

17. Kawai S, Amano A. BRCA1 regulates microRNA biogenesis via the DROSHA microprocessor complex. J Cell Biol 2012; 197: 201-208.

18. Suzuki HI, Yamagata K, Sugimoto K, Iwamoto T, Kato S, Miyazono K. Modulation of microRNA processing by p53. Nature 2009; 460: 529-533.

19. Yan M, Zhang Y, He B, Xiang J, Wang ZF, Zheng FM et al. IKKalpha restoration via EZH2 suppression induces nasopharyngeal carcinoma differentiation. Nat Commun 2014; 5: 3661.

20. Deng L, Li Y, Ai P, Xie Y, Zhu H, Chen N. Increase in IkappaB kinase alpha expression suppresses the tumor progression and improves the prognosis for nasopharyngeal carcinoma. Mol Carcinog 2013; 54: 156-165.

21. Tang $X$, Zhang $Y$, Tucker L, Ramratnam B. Phosphorylation of the RNase III enzyme Drosha at Serine300 or Serine302 is required for its nuclear localization. Nucleic Acids Res 2010; 38: 6610-6619.

22. Besse A, Sana J, Fadrus P, Slaby O. MicroRNAs involved in chemo- and radioresistance of high-grade gliomas. Tumour Biol 2013; 34: 1969-1978.

23. Czochor JR, Glazer PM. microRNAs in cancer cell response to ionizing radiation. Antioxid Redox Signal 2014; 21: 293-312.

24. Carthew RW, Sontheimer EJ. Origins and mechanisms of miRNAs and siRNAs. Cell 2009; 136: $642-655$

25. Kim VN, Han J, Siomi MC. Biogenesis of small RNAs in animals. Nat Rev Mol Cell Biol 2009; 10: 126-139.

26. Ling H, Fabbri M, Calin GA. MicroRNAs and other non-coding RNAs as targets for anticancer drug development. Nat Rev Drug Discov 2013; 12: 847-865.

27. Gregory RI, Yan KP, Amuthan G, Chendrimada T, Doratotaj B, Cooch $\mathrm{N}$ et al. The microprocessor complex mediates the genesis of microRNAs. Nature 2004; 432: 235-240.
28. Morlando M, Ballarino M, Gromak N, Pagano F, Bozzoni I, Proudfoot NJ. Primary microRNA transcripts are processed co-transcriptionally. Nat Struct Mol Biol 2008; 15: 902-909.

29. Han J, Lee Y, Yeom KH, Kim YK, Jin H, Kim VN. The Drosha-DGCR8 complex in primary microRNA processing. Genes Dev 2004; 18: 3016-3027.

30. Michlewski G, Guil S, Semple CA, Caceres JF. Posttranscriptional regulation of miRNAs harboring conserved terminal loops. Mol Cell 2008; 32: 383-393.

31. Viswanathan SR, Daley GQ, Gregory RI. Selective blockade of microRNA processing by Lin28. Science 2008; 320: 97-100.

32. Davis BN, Hilyard AC, Nguyen PH, Lagna G, Hata A. Smad proteins bind a conserved RNA sequence to promote microRNA maturation by Drosha. Mol Cell 2010; 39 : 373-384.

33. Anest V, Hanson JL, Cogswell PC, Steinbrecher KA, Strahl BD, Baldwin AS. A nucleosomal function for IkB kinase-a in NF-kB-dependent gene expression. Nature 2003; 423: 659-663.

34. Yamamoto Y, Verma UN, Prajapati S, Kwak YT, Gaynor RB. Histone H3 phosphorylation by IKKa is critical for cytokine-induced gene expression. Nature 2003; 423: 655-659.

35. Park KJ, Krishnan V, O'Malley BW, Yamamoto Y, Gaynor RB. Formation of an IKKalphadependent transcription complex is required for estrogen receptor-mediated gene activation. Mol Cell 2005; 18: 71-82.

36. Chen C, Zhang Y, Zhang L, Weakley SM, Yao Q. MicroRNA-196: critical roles and clinical applications in development and cancer. J Cell Mol Med 2011; 15: 14-23.

37. Schimanski CC, Frerichs K, Rahman F, Berger M, Lang H, Galle PR et al. High miR-196a levels promote the oncogenic phenotype of colorectal cancer cells. World J Gastroenterol 2009; 15: 2089-2096.

38. Wang $Y X$, Zhang XY, Zhang BF, Yang $C Q$, Chen XM, Gao HJ. Initial study of microRNA expression profiles of colonic cancer without lymph node metastasis. J Dig Dis 2010; 11: 50-54.

39. Braig S, Mueller DW, Rothhammer T, Bosserhoff AK. MicroRNA miR-196a is a central regulator of HOX-B7 and BMP4 expression in malignant melanoma. Cell Mol Life Sci 2010; 67: 3535-3548

40. Li Y, Zhang M, Chen H, Dong Z, Ganapathy V, Thangaraju M et al. Ratio of miR-196 s to HOXC8 messenger RNA correlates with breast cancer cell migration and metastasis. Cancer Res 2010; 70: 7894-7904.

41. Kim YJ, Bae SW, Yu SS, Bae YC, Jung JS. miR-196a regulates proliferation and osteogenic differentiation in mesenchymal stem cells derived from human adipose tissue. J Bone Miner Res 2009; 24: 816-825.

42. Cheung ST, Huang DP, Hui AB, Lo KW, Ko CW, Tsang YS et al. Nasopharyngeal carcinoma cell line (C666-1) consistently harbouring Epstein-Barr virus. Int J Cancer 1999; 83: 121-126.

43. Glaser R, Zhang HY, Yao KT, Zhu HC, Wang FX, Li GY et al. Two epithelial tumor cell lines (HNE-1 and HONE-1) latently infected with Epstein-Barr virus that were derived from nasopharyngeal carcinomas. Proc Natl Acad Sci USA 1989; 86: 9524-9528.

44. Lo AK, Liu Y, Wang XH, Huang DP, Yuen PW, Wong YC et al. Alterations of biologic properties and gene expression in nasopharyngeal epithelial cells by the Epstein-Barr virusencoded latent membrane protein 1. Lab Invest 2003; 83: 697-709.

45. To EW, Chan KC, Leung SF, Chan LY, To KF, Chan AT et al. Rapid clearance of plasma Epstein-Barr virus DNA after surgical treatment of nasopharyngeal carcinoma. Clin Cancer Res 2003; 9: 3254-3259.

46. Shanmugaratnam K, Sobin LH. The World Health Organization histological classification of tumours of the upper respiratory tract and ear. A commentary on the second edition. Cancer 1993; 71: 2689-2697.

47. Feng XP, Yi H, Li MY, Li XH, Yi B, Zhang PF et al. Identification of biomarkers for predicting nasopharyngeal carcinoma response to radiotherapy by proteomics. Cancer Res 2010; 70: $3450-3462$.

\section{Supplementary Information accompanies this paper on Cell Death and Differentiation website (http://www.nature.com/cdd)}

